\title{
Theobromine consumption does not improve fasting and postprandial vascular function in overweight and obese subjects
}

\author{
Lotte Smolders $^{1} \cdot$ Ronald P. Mensink ${ }^{1} \cdot$ Jose J. van den Driessche ${ }^{1} \cdot$ Peter J. Joris ${ }^{1} \cdot$ Jogchum Plat $^{1}$
}

Received: 10 October 2017 / Accepted: 6 January 2018 / Published online: 12 January 2018

(c) The Author(s) 2018. This article is an open access publication

\begin{abstract}
Backgound Theobromine, a component of cocoa, may favorably affect conventional lipid-related cardiovascular risk markers, but effects on flow-mediated dilation (FMD) and other vascular function markers are not known.

Objective To evaluate the effects of 4-week theobromine consumption $(500 \mathrm{mg} /$ day $)$ on fasting and postprandial vascular function markers.

Design In a randomized, double-blind crossover study, 44 apparently healthy overweight $(N=30)$ and obese $(N=14)$ men and women with low HDL-C concentrations, consumed daily $500 \mathrm{mg}$ theobromine or placebo for 4 weeks. After 4 weeks, FMD, peripheral arterial tonometry (PAT), augmentation index (AIx), pulse wave velocity (PWV), blood pressure (BP) and retinal microvasculature measurements were performed. These measurements were carried out under fasting conditions and $2.5 \mathrm{~h}$ after a high-fat mixed meal challenge.

Results 4-week theobromine consumption did not change fasting vascular function markers, except for a decrease in central AIx (cAIx, $-1.7 \mathrm{pp}, P=0.037)$ and a trend towards smaller venular calibers $(-2 \mu \mathrm{m}, P=0.074)$. Consuming a high-fat mixed meal decreased FMD ( $0.89 \mathrm{pp}, P=0.002)$, reactive hyperemia index (RHI, $-0.30, P<0.001$ ), peripheral systolic BP (SBP, $-3 \mathrm{mmHg}, P \leq 0.001)$, peripheral diastolic BP (DBP, $-2 \mathrm{mmHg}, P \leq 0.001)$, central SBP $(-6 \mathrm{mmHg}, P \leq 0.001)$ and central DBP $(-2 \mathrm{mmHg}, P \leq 0.001)$, but increased heart rate (HR, $2 \mathrm{bpm}, P<0.001)$. Theobromine did not modify these postprandial effects, but increased postprandially the brachial artery diameter $(0.03 \mathrm{~cm}, P=0.015)$, and decreased the cAIx corrected for a HR of 75 (cAIx75, $-5.0 \mathrm{pp}, P=0.004$ ) and peripheral AIx (pAIx, $-6.3 \mathrm{pp}, P=0.017$ ).

Conclusion Theobromine consumption did not improve fasting and postprandial endothelial function, but increased postprandial peripheral arterial diameters and decreased the AIx. These findings do not suggest that theobromine alone contributes to the proposed cardioprotective effects of cocoa.

This trial was registered on clinicaltrials.gov under study number NCT02209025.
\end{abstract}

Keywords Theobromine $\cdot$ Endothelial function $\cdot$ Arterial stiffness $\cdot$ Microvasculature $\cdot$ Postprandial

\section{Abbreviations}

AIx Augmentation index

AVR Arteriolar to venular ratio

BP Blood pressure

Electronic supplementary material The online version of this article (https://doi.org/10.1007/s00394-018-1612-6) contains supplementary material, which is available to authorized users.

Jogchum Plat

j.plat@maastrichtuniversity.nl

1 Department of Human Biology and Movement Sciences, School of Nutrition and Translational Research in Metabolism (NUTRIM), Maastricht University Medical Center, Universiteitssingel 50, P.O. Box 616, 6200 MD Maastricht, The Netherlands
cAIx Central augmentation index

cAIx75 cAIx adjusted for a heart rate of 75

cDBP Central diastolic blood pressure

CRAE Central retinal arteriolar equivalent

CRVE Central venular arteriolar equivalent

cSBP Central systolic blood pressure

CVD Cardiovascular disease

FMD Flow-mediated dilation

HDL-C High-density lipoprotein cholesterol

HR Heart rate

LDL-C Low density lipoprotein cholesterol

NO Nitric oxide

pAIx Peripheral augmentation index

PAT Peripheral arterial tonometry

pDBP Peripheral diastolic blood pressure 


$\begin{array}{ll}\text { pp } & \text { Percentage point } \\ \text { pSBP } & \text { Peripheral systolic blood pressure } \\ \text { PWV } & \text { Pulse wave velocity } \\ \text { PWVcf } & \text { Carotid-femoral PWV } \\ \text { PWVcr } & \text { Carotid-radial PWV } \\ \text { RHI } & \text { Reactive hyperemia index }\end{array}$

\section{Introduction}

Chocolate consumption is associated with a lower risk for cardiovascular diseases (CVD) [1]. These effects are specifically evident for dark chocolate, which has been shown to improve serum lipid profiles [2,3], to reduce blood pressure $[2,4]$, to increase insulin sensitivity, and to improve vascular endothelial function as measured with flow-mediated dilation (FMD) [2]. The components from cocoa responsible for the potentially beneficial effects on FMD are unknown, but it can be argued that theobromine $(500-700 \mathrm{mg} / 100 \mathrm{~g}$ dark chocolate) contributes to these effects. In fact, the acute consumption of low amounts of theobromine $(111 \mathrm{mg})$ and caffeine $(11 \mathrm{mg})$ potentiated the protective effect of cocoa flavanols on postprandial FMD [5]. Furthermore, a 4-week study showed that daily consumption of 500 or $850 \mathrm{mg}$ theobromine lowered fasting low-density lipoprotein cholesterol (LDL-C) and apolipoprotein B (apoB) concentrations, and increased those of serum HDL-C [6, 7] and apoA-I [6]. In contrast to these studies, we recently reported that we could not fully confirm the findings on apoA-I concentrations [7]. Moreover, we observed no effects of a daily theobromine consumption of $500 \mathrm{mg}$ on postprandial lipid responses, while postprandial free fatty acid, glucose and insulin responses were increased [7]. It is known that both postprandial hyperlipidemia [8] and hyperglycemia [9] impair vascular function. Therefore, it is relevant to examine effects of theobromine, not only on fasting vascular function, but also on vascular resilience after a meal challenge.

FMD is a non-invasive vascular function marker to assess endothelial function and an accepted predictive biomarker for future CVD events [10]. Another method to evaluate endothelial function is peripheral arterial tonometry (PAT), which measures the reactive hyperaemia index (RHI) of the small arteries and is negatively correlated with the presence of CVD risk factors [11]. Furthermore, several non-invasive markers exist to assess arterial stiffness such as carotidfemoral pulse wave velocity (PWVcf) and the augmentation index (AIx). PWVcf is associated with a higher frequency of stroke, CVD and total mortality [12], while the AIx is associated with higher CVD risk [13]. Finally, the microvasculature can be studied by evaluating the caliber of the blood vessels in the retina. Cross-sectional wider venules and narrower arterioles predict an increased risk of CVD events in women, but not in men [14].
In agreement with effects on FMD [2], dark chocolate consumption also improved RHI [15]. However, effects on measures of arterial stiffness are conflicting. In one study, no effects were found on PWVcf [13], while in another study beneficial effects were found on Aix [16, 17]. Furthermore, Terai et al. showed no differences in arteriolar and venular width after short-term dark chocolate consumption [12].

So far, long-term effects of theobromine consumption on a wide panel of vascular function markers have never been studied. Therefore, we examined the effects of 4-week theobromine consumption ( $500 \mathrm{mg} /$ day) on FMD, RHI, pulse wave velocity (PWV), AIx, and retinal microvasculature in fasting conditions and after a high-fat meal challenge.

\section{Materials and methods}

\section{Study population}

Details of this study have been published before [7]. Briefly, 44 healthy overweight (BMI $25-30 \mathrm{~kg} / \mathrm{m}^{2} ; n=30$ ) or slightly obese (BMI $30-35 \mathrm{~kg} / \mathrm{m}^{2} ; n=14$ ) men (45-70 years; $n=28$ ) and women (50-70 years; $n=16)$ participated. During two screening visits, with an interval of $\geq 1$-week, blood pressure was measured in fourfold using an Omron M7 (Omron Healthycare Europe B.V., Hoofddorp, the Netherlands). The first measurement was discarded and the last three measurements were averaged. Furthermore, a fasting blood sample was taken for analysis of serum total cholesterol, HDL-C, and plasma glucose concentrations. Inclusion criteria were: fasting serum HDL-C concentrations below the 50th percentile of the Dutch population $(<1.2 \mathrm{mmol} / \mathrm{L}$ for men and $<1.5 \mathrm{mmol} / \mathrm{L}$ for women) [18], fasting serum total cholesterol concentrations $<8.0 \mathrm{mmol} / \mathrm{L}$, fasting plasma glucose concentrations $<7.0 \mathrm{mmol} / \mathrm{L}$, and no use of lipid-lowering, anti-diabetic or anti-hypertensive medication or a medically prescribed diet. All participants gave their written informed consent before entering the study. This study was conducted according to the guidelines laid down in the Declaration of Helsinki. The study protocol was approved by the Medical Ethical Committee of the University Hospital Maastricht. The trail was registered at clinicaltrials.gov under study number NCT02209025.

\section{Study design and product}

This study with a randomized, double-blind, cross-over design consisted of two intervention periods of 4-week separated by a 4 -week washout period. Starting 2 -week before the first intervention period and during the entire study, participants were instructed by a research dietician to avoid cocoa-containing products, for which they received a detailed list with products. Since theobromine is 
a metabolite of caffeine, the consumption of caffeine-containing drinks was restricted to a maximum of four cups a day and volunteers were instructed not to change their intake throughout the study. Subjects consumed in random order a drink $(20 \mathrm{~mL})$ enriched with theobromine $(500 \mathrm{mg} /$ day) or placebo every day during breakfast (Supplemental Table 1). Theobromine was obtained from Fagron (Uitgeest, the Netherlands) and drinks were produced and provided by Pharmavize (Mariakerke, Belgium).

\section{Test day and test meal}

At the end of the 4-week intervention and placebo periods, subjects visited the University in fasting condition (no food or drinks, except water, 12-h before the visit). To minimize effects of the previous meal, we provided all subjects with the same commercially available lasagne $(638 \mathrm{kcal}, 28.4 \mathrm{~g}$ protein, $44.0 \mathrm{~g}$ carbohydrates and $37.6 \mathrm{~g}$ fat) the evening before each of the two test days. Furthermore, subjects were asked to avoid alcohol consumptions and strenuous activities 48 -h before a visit.

In the morning, volunteers arrived at the Department by public transport or car, to standardize measurements as much as possible. Upon arrival and after a 10 min rest in supine position, vascular function measurements were performed in fasting conditions. Next, subjects were asked to consume a high-fat mixed meal $(965 \mathrm{kcal}, 17.9 \mathrm{~g}$ protein, $86.7 \mathrm{~g}$ carbohydrates, $60.6 \mathrm{~g}$ fat and $341 \mathrm{mg}$ cholesterol), which was actually a shake prepared with regular food items bought in the local supermarket, together with their experimental drink, within $10 \mathrm{~min}$. For the next 2.5 -h following the meal, participants were not allowed to eat or drink anything except water. After 2.5-h (T150), the same panel of vascular function measurements was performed in the same order, using the same protocols.

\section{Vascular measurements}

Investigators were blinded during the study and data analyses. All vascular measurements were performed in a quiet and temperature-controlled $\left(22{ }^{\circ} \mathrm{C}\right)$ room. Peripheral systolic blood pressure (pSBP), peripheral diastolic blood pressure (pDBP), FMD, RHI, PWVcf, AIx and retinal vascular image measurements were determined as described before. Furthermore, carotid-radial PWV (PWV cr) was measured with the SphygmoCor, as described for the PWVcf [19]. Central systolic blood pressure (cSBP) and central diastolic blood pressure (cDBP) values were obtained from the SphygmoCor measurements.

\section{Statistical analysis}

Before the start of the study, it was calculated that the statistical power to detect a true difference of at least $1.20 \%$ points (pp) in FMD between the experimental and control period was over $80 \%(\alpha=0.05)$, when 43 subjects successfully completed the study. For these calculations, a withinsubject variability of $2.82 \mathrm{pp}$ in FMD [20] was used. As the expected drop-out rate was $10 \%$, the aim was to recruit 48 men and women.

All data is presented as mean \pm SD unless indicated otherwise. All parameters were checked for normal distributions with the Shapiro-Wilk test. Fasting measurements after 4-week of placebo or theobromine intervention were compared with the general mixed model procedure with subject as random factor, and treatment and period as fixed factors. Differences in postprandial changes after 4 weeks of placebo or theobromine interventions were also evaluated with general mixed models with subject as random factor and treatment and meal as fixed factors and a treatment $\times$ meal interaction. If this treatment $\times$ meal interaction was not significant, it was omitted from the model. Results were considered statistically significant if $P \leq 0.05$. All statistical analyses were performed using SPSS 20.0 for Mac (SPSS Inc., Chicago, IL, USA).

\section{Results}

\section{Study participants}

After screening, 48 subjects were eligible for participation and started the study. During the first intervention period, four participants (one male and three female) discontinued the study. Thus, 44 participants completed the study. The flow diagram and subject characteristic are presented in supplemental Fig. 1 and supplemental Table 2. RHI data was missing for three persons, due to technical problems. For two subjects (one man, one woman), T0 values were missing and for one male participant, a T150 value was absent. For four persons CRAE and CRVE calibers were missing (one man and three women) and for two persons (one man and one woman) the T150 values were missing, because of a poor quality of the fundus photos.

\section{Fasting vascular function}

In the fasting condition, theobromine consumption did not change FMD, brachial artery diameter, and RHI. Furthermore, PWVcf, PWVcr, pAIx and cAIx75 did not change, but the cAIx was significantly lower after theobromine intake $(-1.7 \mathrm{pp}, 95 \% \mathrm{CI}-6.1,-0.2, P=0.037)$. The CRAE and AVR also remained stable during the study, but theobromine 
intake tended to decrease the CRVE $(-2 \mu \mathrm{m}, 95 \% \mathrm{CI}-4$, $0, P=0.074)$. Finally, fasting BP and HR were not affected (Table 1).

\section{Postprandial vascular function}

As expected, high-fat mixed meal intake significantly decreased FMD $(-0.89 \mathrm{pp}, 95 \%$ CI $-1.43,-0.35$, $P=0.002)$ and RHI $(-0.30,95 \%$ CI $-0.43,-0.16$, $P<0.001)$ responses, but these effects did not depend on theobromine consumption. However, the brachial artery diameter increased when theobromine was part of the test meal $(0.03 \mathrm{~cm}, 95 \%$ CI $1.23,4.45, P=0.015$ for treatment $\times$ meal effects).

Test meal consumption did not affect arterial stiffness as measured via PWVcf and PWVcr. These effects were not changed by theobromine consumption. However, theobromine consumption tended to decrease the postprandial cAIx $(-4.9 \mathrm{pp}, 95 \% \mathrm{CI}-5.8,-0.7, P=0.080$ for treatment $\times$ meal effects $)$ and decreased the pAIx $(-6.3 \mathrm{pp}$,
95\% CI $-9.2,-2.4, P=0.017$ for treatment $\times$ meal effects) and cAIx75 $(-5.0 \mathrm{pp}, 95 \% \mathrm{CI}-6.8,-2.4, P=0.004$ for treatment $\times$ meal effects). Test meal consumption did not change retinal vascular calibers. Effects were not changed by theobromine consumption. Finally, the test meal significantly decreased cSBP $(-5 \mathrm{mmHg}, 95 \% \mathrm{CI}-8,-4$, $P \leq 0.001)$, cDBP $(-2 \mathrm{mmHg}, 95 \% \mathrm{CI}-3,-1, P \leq 0.001)$, pSBP $(-3 \mathrm{mmHg}, 95 \% \mathrm{CI}-5,-1, P \leq 0.001)$ and $\mathrm{pDBP}$ $(-2 \mathrm{mmHg}, 95 \% \mathrm{CI}-3,-1, P \leq 0.001)$ and increased HR (2 bpm, 95\% CI 1, 3, $P \leq 0.001$ ). These effects were not modified by theobromine (Table 1 ).

\section{Discussion}

This randomized, double-blind, placebo-controlled intervention study showed that a daily intake of $500 \mathrm{mg}$ theobromine for 4 weeks did not affect FMD, RHI, PWV and the retinal microvasculature in fasting and postprandial conditions. However, theobromine consumption increased brachial

Table 1 Brachial diameter, brachial artery FMD, RHI, PWVcf, PWVcr, cAIx, cAIx75, pAIx, CRAE, CRVE, AVR, pSBP, pDBP, cSBP, cDBP and HR in fasting (T0) and postprandial (T150) condition after 4 weeks of placebo or theobromine consumption

\begin{tabular}{|c|c|c|c|c|c|c|}
\hline & \multicolumn{3}{|l|}{ Placebo } & \multicolumn{3}{|l|}{ Theobromine } \\
\hline & T0 & $\mathrm{T} 150$ & Change & T0 & $\mathrm{T} 150$ & Change \\
\hline Brachial diameter $(\mathrm{cm})$ & $0.49 \pm 0.06$ & $0.50 \pm 0.08$ & $0.00 \pm 0.04$ & $0.49 \pm 0.07$ & $0.52 \pm 0.08$ & $0.03 \pm 0.04 *$ \\
\hline Brachial artery FMD $(\%)^{\$}$ & $4.87 \pm 2.54$ & $3.87 \pm 2.32$ & $-1.00 \pm 2.97$ & $4.43 \pm 2.01$ & $3.65 \pm 2.25$ & $-0.78 \pm 2.48$ \\
\hline $\mathrm{RHI}^{1, \$}$ & $2.64 \pm 0.68$ & $2.38 \pm 0.61$ & $-0.24 \pm 0.65$ & $2.58 \pm 0.61$ & $2.23 \pm 0.47$ & $-0.35 \pm 0.60$ \\
\hline $\operatorname{PWVcr}(\mathrm{m} / \mathrm{s})$ & $7.1 \pm 1.1$ & $7.1 \pm 1.1$ & $-0.1 \pm 1.2$ & $7.4 \pm 1.3$ & $7.1 \pm 1.5$ & $-0.3 \pm 1.6$ \\
\hline PWVcf (m/s) & $9.0 \pm 1.4$ & $9.0 \pm 1.6$ & $0.0 \pm 1.3$ & $8.8 \pm 1.6$ & $9.0 \pm 1.5$ & $0.2 \pm 1.5$ \\
\hline cAIx $(\%)$ & $28.3 \pm 9.9$ & $21.9 \pm 10.5$ & $-6.4 \pm 6.2$ & $26.6 \pm 10.4^{\#}$ & $15.2 \pm 11.2$ & $-11.3 \pm 8.4$ \\
\hline pAIx $(\%)$ & $-14.8 \pm 14.9$ & $-24.1 \pm 13.7$ & $-9.3 \pm 10.6$ & $-16.8 \pm 15.3$ & $-32.4 \pm 13.8$ & $-15.6 \pm 14.4^{*}$ \\
\hline cAIx75 (\%) & $21.6 \pm 8.7$ & $16.3 \pm 9.6$ & $-5.3 \pm 6.5$ & $21.4 \pm 9.3$ & $11.2 \pm 10.7$ & $-10.3 \pm 8.2^{*}$ \\
\hline $\operatorname{CRAE}(\mu \mathrm{m})^{3}$ & $135 \pm 19$ & $135 \pm 19$ & $0 \pm 9$ & $134 \pm 19$ & $136 \pm 19$ & $2 \pm 6$ \\
\hline CRVE $(\mu \mathrm{m})^{3}$ & $230 \pm 14$ & $231 \pm 13$ & $0 \pm 5$ & $228 \pm 14$ & $231 \pm 13$ & $2 \pm 7$ \\
\hline $\mathrm{AVR}^{3}$ & $0.59 \pm 0.09$ & $0.59 \pm 0.09$ & $0.00 \pm 0.05$ & $0.58 \pm 0.09$ & $0.59 \pm 0.09$ & $0.01 \pm 0.04$ \\
\hline $\mathrm{pSBP}(\mathrm{mmHg})^{\$}$ & $134 \pm 14$ & $132 \pm 12$ & $-3 \pm 9$ & $134 \pm 14$ & $130 \pm 13$ & $-4 \pm 10$ \\
\hline $\mathrm{pDBP}(\mathrm{mmHg})^{\$}$ & $85 \pm 10$ & $83 \pm 8$ & $-2 \pm 5$ & $86 \pm 10$ & $83 \pm 9$ & $-3 \pm 6$ \\
\hline $\mathrm{cSBP}(\mathrm{mmHg})^{\$}$ & $126 \pm 13$ & $121 \pm 11$ & $-5 \pm 8$ & $125 \pm 12$ & $118 \pm 13$ & $-7 \pm 10$ \\
\hline $\mathrm{cDBP}(\mathrm{mmHg})^{\$}$ & $86 \pm 9$ & $84 \pm 9$ & $-2 \pm 6$ & $87 \pm 9$ & $84 \pm 9$ & $-3 \pm 5$ \\
\hline $\mathrm{HR}(\mathrm{bpm})^{\$}$ & $62 \pm 9$ & $64 \pm 10$ & $2 \pm 4$ & $62 \pm 8$ & $65 \pm 10$ & $3 \pm 7$ \\
\hline
\end{tabular}

Values are mean \pm SD. $n=44$. Linear mixed models were conducted to find significant differences

$F M D$ flow mediated dilation, $R H I$ reactive hyperemia index, $P W V$ pulse wave velocity, $P W V c f$ PWV of the carotis-femoralis, $P W V c r$ PWV of the carotis-radialis, $c A I x$ central augmentation index, $c A I x 75$ cAIx corrected for a heart rate of 75, $p A I x$ peripheral augmentation index, $C R A E$ mean arteriolar width, $C R V E$ mean venular width, $A V R$ arteriolar to venular ratio, $p$ peripheral, $c$ central, $S B P$ systolic blood pressure, $D B P$ diastolic blood pressure, $H R$ heart rate

${ }^{\#} P<0.05$ for fasting differences from placebo

$* P<0.05$ for treatment $\times$ meal effects

${ }^{\$} P<0.05$ for meal effects

${ }^{1} n=42$ at $\mathrm{T} 0, n=41$ at $\mathrm{T} 150$ due to missing values

${ }^{2} n=41$ at $\mathrm{T} 0, n=39$ at $\mathrm{T} 150$ due to missing values 
arterial diameters and decreased the AIx during the postprandial phase.

The amount of $500 \mathrm{mg}$ theobromine provided corresponds to approximately $67-100 \mathrm{~g}$ of dark chocolate [21]. It has been shown that consumption of $100 \mathrm{~g}$ dark chocolate for 15 days increased fasting FMD by $1.5 \mathrm{pp}$ [22], which has been explained by an increase in nitric oxide (NO) concentrations due to a higher endothelial-derived NO synthase activity [23]. Our study was adequately powered to detect such a change. Moreover, the finding that fasting RHI-which is also NO-mediated but more related to the small arteries and the microvasculature-did not change after theobromine consumption was also opposite to the effects observed after consuming cocoa [15]. Furthermore, theobromine did not modify the effects of a meal challenge on vascular resilience. It is well known that a meal high in fat or high in carbohydrates impairs endothelial function $[24,25]$. During postprandial hyperlipemia and hyperglycemia, the production of reactive oxygen species increases, which decreases NO bioavailability and thereby endothelial function [24, 25]. Indeed, also our test meal stressed the endothelium, as evidenced by decreases in postprandial FMD and RHI values, which is in agreement with other studies [26-28]. In contrast to our results, flavanol-rich cocoa consumption ameliorated the decrease in FMD after intake of a fatty meal [29], while flavonoid-rich dark chocolate consumption even increased FMD values $1 \mathrm{~h}$ after intake [30]. Our data, therefore, suggests that the improvement in endothelial function after cocoa consumption is not solely due to the theobromine content of cocoa. Cocoa also contains other bioactive compounds that may affect FMD, such as epicatechin [2]. Furthermore, it is possible that synergistic effects of the different bioactive components in cocoa have caused the beneficial effects on FMD and RHI. Indeed, Sansone et al. have recently shown that a combination of theobromine $(111 \mathrm{mg})$ and caffeine $(11 \mathrm{mg})$ did not change the FMD, while flavanol consumption $(820 \mathrm{mg}$ ) alone increased the FMD. When the flavanols were consumed together with the mixture of theobromine and caffeine, circulating concentrations of flavanol metabolites were increased, while the FMD improved even more [5].

Although theobromine did not change the FMD, we observed an unexpected increase in brachial artery diameters during the postprandial phase. Unfortunately, most studies investigating the effects of cocoa did not report effects on brachial artery diameters. However, one acute study showed an increase in the brachial artery diameter after flavonoid-rich dark chocolate consumption, but with a simultaneous increase in FMD values [30]. We can only speculate about the mechanism underlying the increase in brachial artery diameter. First, theobromine inhibits cyclic adenosinemonophosphate (cAMP)-phosphodiesterase [31, 32], which increases cellular cAMP levels. As a response, intracellular calcium concentrations may decrease, followed by dilatation of the skeletal muscle vasculature [33]. A second potential explanation relates to the increased postprandial insulin responses after theobromine consumption, as we have already earlier reported [7]. Insulin is known to cause vasodilatation of the larger arteries [34], leading to enlarged artery diameters.However, it is also possible that the totality of metabolic changes including elevated insulin, glucose and hsCRP in the postprandial phase after theobromine consumption as reported earlier [7], is the reason underlying the observed effects on the vasculature during the postprandial phase.

Theobromine did not change fasting and postprandial PWV, but decreased fasting and postprandial AIx. This suggests that effects on parameters reflecting arterial stiffness are divergent, as has also been reported in other studies [27, 35]. Differences in PWV are frequently caused by changes in blood pressure [36]. In agreement with the lack of effect on PWV, theobromine did not change fasting and postprandial blood pressure parameters. Neufingerl et al. also observed no effects of 4-week of theobromine consumption on fasting blood pressure [6]. Furthermore, cocoa consumption did not affect postprandial blood pressure [30, 37] and PWVcf values [30]. In contrast, the consumption of theobromineenriched flavonoid-rich cocoa drink for 3 weeks increased fasting blood pressure and postprandial PWVcf, while it decreased postprandial blood pressure in hypertensive patients [38]. Possibly, the difference in theobromine dose and drink composition can explain the discrepancy with our findings, since van den Bogaard et al. used a daily theobromine intake of $979 \mathrm{mg}$, which was consumed in combination with flavonoids provided by the cocoa [38]. Structural characteristics of the vascular wall also determine PWV [39]. However, as both blood pressure and PWV did not change, it can be deduced that these characteristics were also not changed.

Unrelated to theobromine intake, we observed a postprandial decrease in blood pressure, but the anticipated decrease in postprandial PWV was not observed. However, effects of meal consumption on blood pressure as related to PWV are conflicting. One study has reported an increase in blood pressure and PWVcf after meal consumption [40]; another study a decrease in blood pressure, but no change in PWVcf [41], while no change in blood pressure but an increase in PWVcr has also been reported [42]. For now, it is not clear what causes the discrepancy between the different studies, but it may relate to differences in the amounts of fat in the test meals between the studies [40-42].

In our study, theobromine decreased fasting cAIx, but did not change fasting pAIx and cAIx75. This is in contrast with the effects of cocoa, since acute and 4-week dark chocolate consumption decreased fasting cAIx75 $[16,17]$. Except for the effects on fasting AIx, the test meal with theobromine 
decreased cAIx75 and pAIx. This decrease may be related to the postprandial increase in peripheral artery diameters, as a blood vessel with a larger diameter causes a lower reflection wave, leading to a lower AIx. It can, therefore, be argued that our findings suggest that the main effect of theobromine is dilation of the small and medium-sized peripheral arteries in the postprandial state. If true, then it is unclear why the PWVcr-a measure for peripheral vascular stiffness-did not decrease after theobromine with meal consumption. Finally, theobromine and meal consumption did not affect the arteriolar and venular diameters in the fundus vasculature. Also, acute dark chocolate consumption had no effect on postprandial arteriolar and venular calibers [12].

In conclusion, theobromine consumption did not improve fasting and postprandial endothelial function, but increased postprandial peripheral arterial diameters and decreased the AIx. These findings do not suggest that theobromine alone contributes to the proposed cardioprotective effects of cocoa.

Acknowledgements We would like to thank M. Beckers, D. Luiten and $\mathrm{C}$ op ' $\mathrm{t}$ Eyndt for their technical and dietetic assistance throughout the study. This research was supported by the Dutch Technology Foundation STW, which is part of the Netherlands Organisation for Scientific Research (NWO), and which is partly funded by the Ministry of Economic Affairs. Grant Number 11349.

Author contributions Formulating the research question: LS, RPM, JP. Designed the study: LS, RPM, JP. Conducted the study: LS, RPM, JP. Collected data LS. Performed statistical analysis: LS, RPM, JP. Interpreted data: LS, RPM, JP. Wrote manuscript: LS, RPM, JP.

Open Access This article is distributed under the terms of the Creative Commons Attribution 4.0 International License (http://creativecommons.org/licenses/by/4.0/), which permits unrestricted use, distribution, and reproduction in any medium, provided you give appropriate credit to the original author(s) and the source, provide a link to the Creative Commons license, and indicate if changes were made.

\section{References}

1. Kwok CS, Boekholdt SM, Lentjes MA, Loke YK, Luben RN, Yeong JK, Wareham NJ, Myint PK, Khaw KT (2015) Habitual chocolate consumption and risk of cardiovascular disease among healthy men and women. Heart 101:1279-1287

2. Hooper L, Kay C, Abdelhamid A, Kroon PA, Cohn JS, Rimm EB, Cassidy A (2012) Effects of chocolate, cocoa, and flavan-3-ols on cardiovascular health: a systematic review and meta-analysis of randomized trials. Am J Clin Nutr 95:740-51

3. Tokede OA, Gaziano JM, Djousse L (2011) Effects of cocoa products/dark chocolate on serum lipids: a meta-analysis. Eur J Clin Nutr 65:879-86

4. Ried K, Sullivan T, Fakler P, Frank OR, Stocks NP (2010) Does chocolate reduce blood pressure? A meta-analysis. BMC Med 8:39

5. Sansone R, Ottaviani JI, Rodriguez-Mateos A, Heinen Y, Noske D, Spencer JP, Crozier A, Merx MW, Kelm M, Schroeter H et al (2016) Methylxanthines enhance the effects of cocoa flavanols on cardiovascular function: randomized, double-masked controlled studies. Am J Clin Nutr 105:352-360

6. Neufingerl N, Zebregs YE, Schuring EA, Trautwein EA (2013) Effect of cocoa and theobromine consumption on serum HDLcholesterol concentrations: a randomized controlled trial. Am J Clin Nutr 97:1201-1209

7. Smolders L, Mensink RP, Boekschoten MV, de Ridder RJ, Plat J (2017) Theobromine does not affect postprandial lipid metabolism and duodenal gene expression, but has unfavorable effects on postprandial glucose and insulin responses in humans. Clin Nutr. https://doi.org/10.1016/j.clnu.2017.06.007 (Epub ahead of print)

8. Vafeiadou K, Weech M, Sharma V, Yaqoob P, Todd S, Williams CM, Jackson KG, Lovegrove JA (2012) A review of the evidence for the effects of total dietary fat, saturated, monounsaturated and n-6 polyunsaturated fatty acids on vascular function, endothelial progenitor cells and microparticles. Br J Nutr 107:303-24

9. Mah E, Noh SK, Ballard KD, Matos ME, Volek JS, Bruno RS (2011) Postprandial hyperglycemia impairs vascular endothelial function in healthy men by inducing lipid peroxidation and increasing asymmetric dimethylarginine:arginine. J Nutr 141:1961-1968

10. Ras RT, Streppel MT, Draijer R, Zock PL (2013) Flow-mediated dilation and cardiovascular risk prediction: a systematic review with meta-analysis. Int J Cardiol 168:344-51

11. Poredos P, Jezovnik MK (2013) Testing endothelial function and its clinical relevance. J Atheroscler Thromb 20:1-8

12. Terai M, Ohishi M, Ito N, Takagi T, Tatara Y, Kaibe M, Komai $\mathrm{N}$, Rakugi H, Ogihara T (2008) Comparison of arterial functional evaluations as a predictor of cardiovascular events in hypertensive patients: the Non-Invasive Atherosclerotic Evaluation in Hypertension (NOAH) study. Hypertens Res 31:1135-1145

13. Vlachopoulos C, Aznaouridis K, O'Rourke MF, Safar ME, Baou K, Stefanadis C (2010) Prediction of cardiovascular events and all-cause mortality with central haemodynamics: a systematic review and meta-analysis. Eur Heart J 31:1865-1871

14. McGeechan K, Liew G, Macaskill P, Irwig L, Klein R, Klein BE, Wang JJ, Mitchell P, Vingerling JR, de Jong PT et al (2009) Prediction of incident stroke events based on retinal vessel caliber: a systematic review and individual-participant meta-analysis. Am J Epidemiol 170:1323-1332

15. Fisher ND, Hughes M, Gerhard-Herman M, Hollenberg NK (2003) Flavanol-rich cocoa induces nitric-oxide-dependent vasodilation in healthy humans. J Hypertens 21:2281-2286

16. Esser D, Mars M, Oosterink E, Stalmach A, Muller M, Afman LA (2014) Dark chocolate consumption improves leukocyte adhesion factors and vascular function in overweight men. FASEB J 28:1464-1473

17. Pereira T, Maldonado J, Laranjeiro M, Coutinho R, Cardoso E, Andrade I, Conde J (2014) Central arterial hemodynamic effects of dark chocolate ingestion in young healthy people: a randomized and controlled trial. Cardiol Res Pract 2014:945951

18. Bos JHMdV, Wolffenbuttel BHR, Verhagen H, Hillege JL, Feskens EJM (2007) De prevalentie van het metabool syndroom in Nederland: verhoogd risico op hart- en vaatziekten en diabetes mellitus type 2 bij een kwart van de personen jonger dan 60 jaar. Ned Tijdschr Geneeskd 151:2382-2388

19. Joris PJ, Plat J, Kusters YH, Houben AJ, Stehouwer CD, Schalkwijk CG, Mensink RP (2016) Diet-induced weight loss improves not only cardiometabolic risk markers but also markers of vascular function: a randomized controlled trial in abdominally obese men. Am J Clin Nutr 105:23-31

20. De Roos NM, Bots ML, Schouten EG, Katan MB (2003) Withinsubject variability of flow-mediated vasodilation of the brachial artery in healthy men and women: implications for experimental studies. Ultrasound Med Biol 29:401-406 
21. Franco R, Onatibia-Astibia A, Martinez-Pinilla E (2013) Health benefits of methylxanthines in cacao and chocolate. Nutrients 5:4159-4173

22. Grassi D, Desideri G, Necozione S, di Giosia P, Barnabei R, Allegaert L, Bernaert H, Ferri C (2015) Cocoa consumption dosedependently improves flow-mediated dilation and arterial stiffness decreasing blood pressure in healthy individuals. J Hypertens 33:294-303

23. Monahan KD (2012) Effect of cocoa/chocolate ingestion on brachial artery flow-mediated dilation and its relevance to cardiovascular health and disease in humans. Arch Biochem Biophys 527:90-94

24. Kawano H, Motoyama T, Hirashima O, Hirai N, Miyao Y, Sakamoto T, Kugiyama K, Ogawa H, Yasue H (1999) Hyperglycemia rapidly suppresses flow-mediated endothelium-dependent vasodilation of brachial artery. J Am Coll Cardiol 34:146-154

25. Nappo F, Esposito K, Cioffi M, Giugliano G, Molinari AM, Paolisso G, Marfella R, Giugliano D (2002) Postprandial endothelial activation in healthy subjects and in type 2 diabetic patients: role of fat and carbohydrate meals. J Am Coll Cardiol 39:1145-1150

26. Bae JH, Bassenge E, Kim KB, Kim YN, Kim KS, Lee HJ, Moon KC, Lee MS, Park KY, Schwemmer M (2001) Postprandial hypertriglyceridemia impairs endothelial function by enhanced oxidant stress. Atherosclerosis 155:517-23

27. Joris PJ, Plat J, Bakker SJ, Mensink RP (2016) Long-term magnesium supplementation improves arterial stiffness in overweight and obese adults: results of a randomized, double-blind, placebocontrolled intervention trial. Am J Clin Nutr 103:1260-1266

28. Ong PJ, Dean TS, Hayward CS, Della Monica PL, Sanders TA, Collins P (1999) Effect of fat and carbohydrate consumption on endothelial function. Lancet 354:2134

29. Westphal S, Luley C (2011) Flavanol-rich cocoa ameliorates lipemia-induced endothelial dysfunction. Heart Vessels 26:511-515

30. Vlachopoulos C, Aznaouridis K, Alexopoulos N, Economou E, Andreadou I, Stefanadis C (2005) Effect of dark chocolate on arterial function in healthy individuals. Am J Hypertens 18:785-791

31. Rasouli M, Zahraie M (2006) Suppression of VLDL associated triacylglycerol secretion by both alpha- and beta-adrenoceptor agonists in isolated rat hepatocytes. Eur J Pharmacol 545:109-14

32. Sugimoto N, Miwa S, Hitomi Y, Nakamura H, Tsuchiya H, Yachie A (2014) Theobromine, the primary methylxanthine found in Theobroma cacao, prevents malignant glioblastoma proliferation by negatively regulating phosphodiesterase-4, extracellular signalregulated kinase, Akt/mammalian target of rapamycin kinase, and nuclear factor-kappa B. Nutr Cancer 66:419-423

33. Hellsten Y, Nyberg M, Jensen LG, Mortensen SP (2012) Vasodilator interactions in skeletal muscle blood flow regulation. J Physiol 590:6297-305

34. Westerbacka J, Wilkinson I, Cockcroft J, Utriainen T, Vehkavaara S, Yki-Jarvinen H (1999) Diminished wave reflection in the aorta. A novel physiological action of insulin on large blood vessels. Hypertension 33:1118-1122

35. Kelly RP, Millasseau SC, Ritter JM, Chowienczyk PJ (2001) Vasoactive drugs influence aortic augmentation index independently of pulse-wave velocity in healthy men. Hypertension 37:1429-1433

36. Cecelja M, Chowienczyk P (2009) Dissociation of aortic pulse wave velocity with risk factors for cardiovascular disease other than hypertension: a systematic review. Hypertension 54:1328-1336

37. Basu A, Betts NM, Leyva MJ, Fu D, Aston CE, Lyons TJ (2015) Acute cocoa supplementation increases postprandial HDL cholesterol and insulin in obese adults with type 2 diabetes after consumption of a high-fat breakfast. J Nutr 145:2325-2332

38. van den Bogaard B, Draijer R, Westerhof BE, van den Meiracker AH, van Montfrans GA, van den Born BJ (2010) Effects on peripheral and central blood pressure of cocoa with natural or high-dose theobromine: a randomized, double-blind crossover trial. Hypertension 56:839-46

39. Laurent S, Cockcroft J, Van Bortel L, Boutouyrie P, Giannattasio C, Hayoz D, Pannier B, Vlachopoulos C, Wilkinson I, StruijkerBoudier H (2006) Expert consensus document on arterial stiffness: methodological issues and clinical applications. Eur Heart J 27:2588-605

40. Lithander FE, Herlihy LK, Walsh DM, Burke E, Crowley V, Mahmud A (2013) Postprandial effect of dietary fat quantity and quality on arterial stiffness and wave reflection: a randomised controlled trial. Nutr J 12:93

41. Murray T, Yang EY, Brunner G, Kumar A, Lakkis N, Misra A, Virani SS, Hartley CJ, Morrisett JD, Ballantyne CM et al (2015) Postprandial effects on arterial stiffness parameters in healthy young adults. Vasc Med 20:501-508

42. Clegg M, McClean C, Davison WG, Murphy HM, Trinick T, Duly E, McLaughlin J, Fogarty M, Shafat A (2007) Exercise and postprandial lipaemia: effects on peripheral vascular function, oxidative stress and gastrointestinal transit. Lipids Health Dis 6:30 